\title{
Biobased Latexes from Soy and Vanillin Derived Vinyl Monomers
}

\author{
Yehor Polunin ${ }^{1}$, Eric Serum ${ }^{2}$, Mukund Sibi $^{2}$, Andriy Voronov ${ }^{1}$ \\ ${ }^{1}$ North Dakota State University, Coatings and Polymeric Materials Department, Fargo, ND, USA \\ yehor.polunin@ndsu.edu; andriy.voronov@ndsu.edu \\ ${ }^{2}$ North Dakota State University, Department of Chemistry and Biochemistry, Fargo, ND, USA \\ eric.serum@ndsu.edu; mukund.sibi@ndsu.edu
}

\section{Extended Abstract}

Recently, we reported on synthesis and characterization of new biobased acrylic monomers derived from variety of plant/vegetable oils (soybean, olive, linseed, sunflower, corn, canola etc.) and their application in free radical polymerization including emulsion/miniemulsion latex synthesis, with a goal to replace petroleum-based constituents and reduce VOC content. ${ }^{1,2}$ Copolymerization study on plant oil-based monomers (POBMs) with a variety of petroleum-based commodity comonomers demonstrated that incorporation of POBM fragments into macromolecular structure provides internal plasticization and hydrophobization, improves toughness of crossilinked latex polymers, enhances adhesion properties and promotes biodegradability of resulted biobased latexes. However, excessive polymers softening was observed when POBM content in the resulted copolymers exceeded $60 \mathrm{wt} \%$, thus making latexes "too soft" and not applicable for film preparation. To be able to further increase biobased content in latexes without worsening the material properties and performance, biobased aromatic comonomers have to be considered for copolymerization with POBM. We hypothesize that combination of a benzene ring (present in the structure of aromatic monomer) and long fatty acid fragments (present in POBMs) provides a lot of opportunities in terms of tuning latex copolymers properties and performance. To this end, vinyl monomer derived from aromatic cardanol (CBM) and monomer from high oleic soybean oil (HOSBM) were successfully copolymerized in miniemulsion to yield biobased latexes in our recent study. ${ }^{3}$ As a result, both biobased fragments contributed to the mechanical properties of the synthesized latexes. The incorporation of aromatic fragments of cardanol enhances Young's modulus of the films, whereas the fatty POBM constituents made the material more flexible. Already $10 \mathrm{wt}$. \% of CBM fragments provided noticeable strength to the soft latex polymeric material from entirely HOSBM. ${ }^{3}$

Furthermore, we elaborated on hypothesis of combining aromatic and aliphatic biobased ingredients in emulsion/miniemulsion copolymerization process. For this purpose, new vanillin-derived compound, 3-allyl-5vinylveratrole $(\mathrm{AVV})^{4}$ was chosen as a comonomer for copolymerization with HOSBM. Having chemical structure similar to petroleum-based commodity vinyl monomer styrene, the AVV can be considered as styrene's biobased alternative when incorporated in a variety of polymer materials. Incorporation of AVV fragments into latex copolymers could balance mechanical properties of the resulted macromolecules by adding the materials strength and enhancing toughness. Additional interesting aspect can be added by presence of allyl bond in the AVV chemical structure which sets task to investigate its polymerizability in free radical mechanism, specifically, whether the allyl group remains retained during the reaction and can be used for post-polymerization cross-linking.

The obtained results show that AVV successfully undergoes free radical homopolymerization $\left(\mathrm{M}_{\mathrm{n}}=2 \quad 100000\right)$ and chain copolymerization. The DSC of polyAVV showed that the synthesized biobased material possesses a $T_{g}$ in the region of $65-70^{\circ} \mathrm{C}$. Finally, feasibility of AVV and HOSBM to interact in chain copolymerization was demonstrated. Both fragments contribute to the properties of resulted latex copolymers. If compared to HOSBM copolymerization with CBM, significantly higher molecular weight copolymers can be obtained. This can be explained by the fact that pronounced for more unsaturated CBM allylic termination, thus chain propagation coexisting with effective chain transfer, did not take place when HOSBM is copolymerized with AVV. As determined by ${ }^{1} \mathrm{H}$ NMR spectroscopy, AVV's allylic group remains unreacted under polymerization reaction conditions used in this study. 


\section{References}

[1] Zoriana Demchuk, Oleg Shevchuk, Ihor Tarnavchyk, Vasylyna Kirianchuk, Ananiy Kohut, Stanislav Voronov, and Andriy Voronov, "Free Radical Polymerization Behavior of the Vinyl Monomers from Plant Oil Triglycerides,” ACS Sustainable Chemistry \& Engineering 20164 (12), 6974-6980

[2] Zoriana Demchuk, Oleh Shevchuk, Ihor Tarnavchyk, Vasylyna Kirianchuk, Maria Lorenson, Ananiy Kohut, Stanislav Voronov, and Andriy Voronov, "Free-Radical Copolymerization Behavior of Plant-Oil-Based Vinyl Monomers and Their Feasibility in Latex Synthesis," ACS Omega 20161 (6), 1374-1382

[3] Zoriana Demchuk, W. S. Jennifer Li, Hermella Eshete, Sylvain Caillol, and Andriy Voronov, "Synergistic Effects of Cardanol- and High Oleic Soybean Oil Vinyl Monomers in Miniemulsion Polymers," ACS Sustainable Chemistry \& Engineering 20197 (10), 9613-9621

[4] Arvin Z. Yu, Eric M. Serum, Anna C. Renner, Jonas M. Sahouani, Mukund P. Sibi, and Dean C. Webster, "Renewable Reactive Diluents as Practical Styrene Replacements in Biobased Vinyl Ester Thermosets" ACS Sustainable Chemistry \& Engineering 20186 (10), 12586-12592. 